\title{
A Proposed Approach to Extend the Economic Order Quantity (EOQ) Model Using Discrete Event Simulation
}

\author{
Giovanni Davoli and Riccardo Melloni \\ Department of Mechanical and Civil Engineering (DIMeC) \\ University of Modena and Reggio Emilia \\ via Vignolese 905, 41100, Modena, Italy \\ giovanni.davoli@unimore.it
}

\begin{abstract}
The economic order quantity (EOQ) and the economic production quantity (EPQ) are well-known and commonly used inventory control techniques. The standard results are easy to apply but are based on a number of unrealistic assumptions. One of the assumption is that the demand is normally distributed in any interval. In several practical cases the assumption about independence of successive demands, and consequently demand normal distribution in any interval, is not supported by real data. This paper investigates the effects on the expected service level (SL) after relaxing normal distribution assumption on the demand. The present work shows a possible strategy to use classic inventory model, such as EOQ/EPQ model, adopting discrete event simulation analysis to quantify model performances under relaxed assumptions.
\end{abstract}

Keywords: EOQ, inventory techniques, discrete event, simulation analysis.

\section{Introduction}

The economic order quantity (EOQ), first introduced by Harris [1], and developed by Brown [2] and Bather [3] with stochastic demand, is a well-known and commonly used inventory control techniques reported in a great variety of hand book, for example: Tersine [4] and Ghiani [5]. The standard EOQ and economic production quantity (EPQ) results are easy to apply but are based on a number of unrealistic assumptions [6]. One of the assumption is that the demand is normally distributed in any interval, it is assumed that successive demands are independent and, consequently, the accumulated demand over many time units is approximately normal. The realization that inventories operate under less than ideal situations gives rise to a subset of inventory modeling theory that performs sensitivity analysis on models operating under stochastic conditions [7]. Several extensions of the classic EOQ/EPQ model have been, Borgonovo [8] presents a good review of them across several fields of research. A branch comprises models where the assumption that all units are of perfect quality is removed, for a deep literature review you can see Chan [9]. Another field of EOQ extended models is focused on deteriorating inventory models for perishables management, for a good review you can see Goyal [10] and Ferguson [11]. Some papers mixed the two proposals to develop a model that better 
fits real practice conditions, for example Inderfurth [12]. Other models modified demands assumptions, for example Chao [13] proposed an EOQ model in which the demand is characterized by a continuous-time Brownian motion process.

Limiting the literature review to nonzero lead time EOQ model, Lowe and Schwarz [14] performed research on the problem where parameters value are not known with certainty and they find that EOQ model is quite insensitive to errors in forecast of demand, their research was extended by Hojati [15] in recent years. Also Dobson [16] studied EOQ model sensitiveness and his results support the previous conclusions. On the other hand Mykytka and Ramberg [17] and later Mahmoud and Younis [18] examine the sensitivity of the EOQ model to errors in forecast of demand and they found that inaccurate estimation produces considerably different results, Higle [19] extends the previous works. There is a good quantity of works about inventory analysis through simulation: Meherez and Ben-Arieh [20] studied a model with stochastic demand and their simulation experiments reveal that the model is not sensitive to demand distribution and parameters accuracy; Naddor [21] states that model results depended on mean and standard deviation of demand, but not on the specific distribution, also Hebert and Deckro [22] results are quite insensitive to demand forecast errors. In contrast Benton [23] states that high measures of demand lumpiness resulted in high service level. Even Lau and Zaki [24] state that EOQ results depends on demand distribution. The various researchers not always agree with one other and the EOQ model sensitivity needs to be more evaluated under stochastic conditions [7]. EOQ model apathy for accurate parameter estimation may have contributed to low usage of the EOQ Ziegler [25]. While some case study reporting EOQ model application can be found, for example De Castro [26], EOQ model low usage and poor results in industrial practice are suggested by recent works in which corrections to the original model are proposed, for example Persona [27].

\section{Purpose}

The purpose of this paper is to investigate the possibility to use discrete event simulation to extend the EOQ model in order to relax some of the standard hypothesis. In several practical cases EOQ assumptions are not supported by real data. The hypothesis about independence of successive demands, and consequently demand normal distribution in any interval, is often not guarantee, for example, when the manufacture uses at the same time different channels selling goods to address the same customers: large scale distribution and traditional retail. In all these cases the total demand in a certain period could fluctuate according with a normal distribution but the successive daily demands are not independent. Moreover, in many practical cases the used time sample to analyze the historical demand is not related to the lead time, often a monthly time sample is used to determinate the standard deviation and the safety stocks (SS) are calculated consequently. In fact, many manufactures experiment that the achieved SL is significantly lower than the SL expected from the theoretical EOQ model. Discrete event simulation can be useful to extend classical model under more realistic hypothesis. An example of the proposed approach is given here where are presented the effects on the expected SL, after relaxing normal distribution and sampling assumptions on the demand. 
Table 1. Symbol and definitions

\begin{tabular}{|c|c|c|}
\hline Symbol & Unit & Definition \\
\hline $\mathbf{D}_{\mathbf{i}}$ & Unit/period & Mean demand per i-period in unit \\
\hline$\sigma_{i}$ & Unit/period & Standard deviation for demand per i-period in unit \\
\hline $\mathbf{d}_{\mathrm{k}}$ & Unit/day & Demand per k-day in unit \\
\hline $\mathbf{N}$ & Day & Number of days for simulation \\
\hline Np & Day & Number of days for period \\
\hline $\mathrm{C}_{\mathbf{l}}$ & Euro/batch & Set-up cost in euro per unit \\
\hline $\mathbf{C}_{\mathrm{s}}$ & Euro/ unit*year & Stock cost in euro per unit per year \\
\hline $\operatorname{Max}_{d}$ & Unit & Maximum demand for a day in unit \\
\hline $\operatorname{Min}_{d}$ & Unit & Minimum demand for a day in unit \\
\hline Lt & Day & Lead time in day \\
\hline$\sigma_{t}$ & Day & Standard deviation for lead time in day \\
\hline EOQ & Unit & Economic production quantity in unit \\
\hline SS & Unit & Safety stocks in unit \\
\hline $\mathbf{S L}_{\mathbf{t}}$ & Rate & $\begin{array}{l}\text { Service level, } 1 \text { minus the ratio between the number } \\
\text { of "stock out" and the number of replenishment orders } \\
\text { issued, while a "stock out" is considered when the } \\
\text { total demand in a lead time exceeds the mean demand } \\
\text { over the lead time. }\end{array}$ \\
\hline SL & Rate & $\begin{array}{l}\text { Service level, } 1 \text { minus the ratio between days of } \\
\text { availability against total days, during the simulated } \\
\text { period }\end{array}$ \\
\hline $\mathbf{S L}_{\mathrm{p}}$ & Rate & $\begin{array}{l}\text { Service level, } 1 \text { minus the ratio between available } \\
\text { units against demanded units, during the simulated } \\
\text { period }\end{array}$ \\
\hline
\end{tabular}

\section{Simulation Model}

This paper is grounded on a discrete events simulation model reproducing a singleitem fixed order quantity system acting under relaxed demand assumption. The model was used to quantify the effectiveness of SS, in term of SL, when SS are calculated according with the EOQ classic model. The notations used in this paper are illustrated in table 1. A set of stochastic functions, provided by SciLab, are used to generate the demand, the simulation model was tested performing normal distributed demand and then this hypothesis is relaxed.

\subsection{Model Validation}

The model validation represents a key aspect of the proposed approach especially because the considered discrete event simulation model is use under stochastic conditions. Simulation outputs are compared to the results provided by the theory, to validate the simulation model in a two steps process. First the behavior in term of stock during time is analyzed, second the achieved SL under stochastic conditions is compared. The first comparison, shown in Fig. 1, is performed for both: the deterministic EOQ model, where the demand is constant, and the stochastic EOQ model, where the demand is normally distributed. The figures shows that the simulation results fit with the standard EOQ theoretical model. 

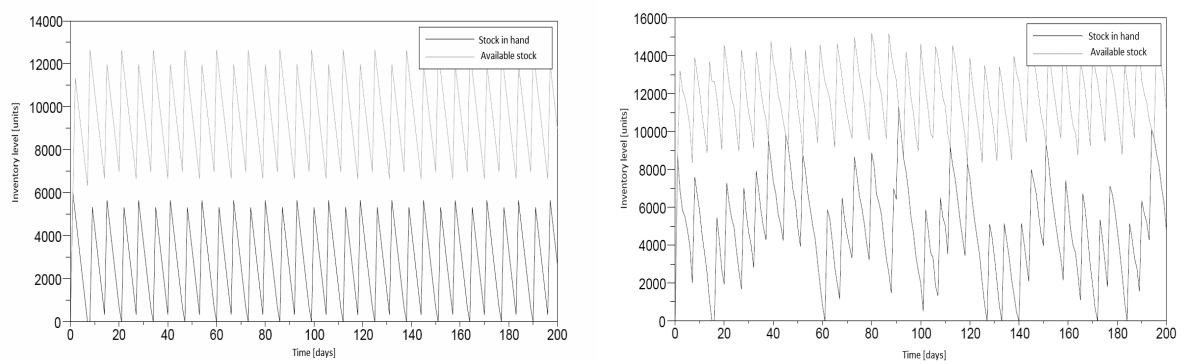

Fig. 1. Inventory level for deterministic (left) and normal distributed (right) demand

The second part of the validation process considers the achieved $\mathrm{SL}_{\mathrm{t}}$ to compare simulation results to theoretical expected values. The SL used in the validation process is named $\mathrm{SL}_{\mathrm{t}}$ defined according with the EOQ theoretical model as: 1 minus the ratio between the number of "stock out" and the number of replenishment orders issued, while a "stock out" is considered when the total demand in a lead time exceeds the mean demand over the lead time. This definition of $\mathrm{SL}_{t}$ is used only to validate the simulation model, while findings are presented in term of redefined $\mathrm{SL}$ and $\mathrm{SL}_{\mathrm{p}}$ in order to have a more relevant definition for industrial practice. This second step of validation process involved only the stochastic model where the demand is normally distributed.
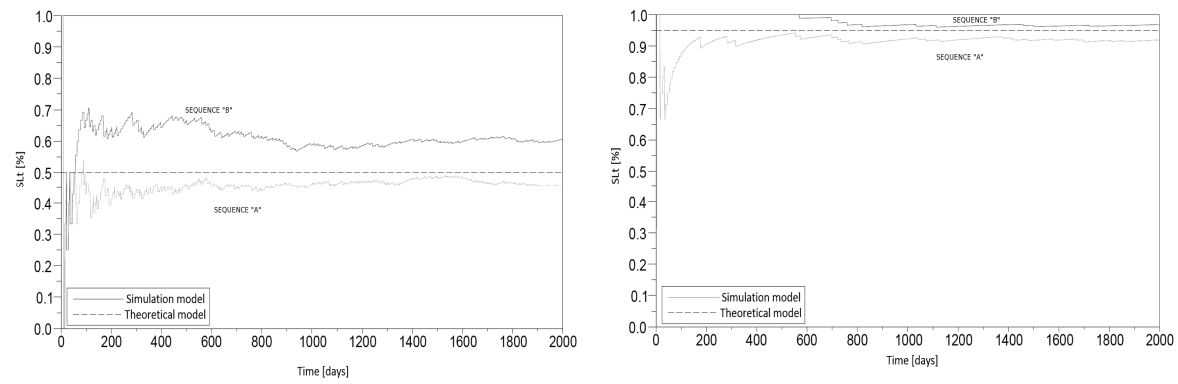

Fig. 2. SLt course for a SL target of 50\% (left) and 95\% (right)

The comparison of $\mathrm{SL}_{t}$ reveals a discretization problem when the model is implemented. In each day the model does these actions:

1. puts the incoming order to stock, increasing the inventory level;

2. fulfills the outgoing order, decreasing the inventory level;

3. checks the inventory level.

The theoretical EOQ model is a mathematical continuous model where all these actions occur at the same time and where an order to refurnish stock is placed when the inventory level reach the reorder point, whatever is the time instant. This continuous behavior could not be implemented in the developed simulation model where the check of the inventory level is done once in a day. The logical sequences used in the simulation model are two: sequence A (pre) and sequence B (post). In sequence $\mathrm{A}$ the simulation code performs, for each day, the actions in this order: 
2-1-3 and this overestimates the inventory level. In sequence B the code performs: 23-1 and this underestimates the inventory level. The validation process considered the $\mathrm{SL}_{\mathrm{t}}$ achieved with SS calculated according with the theoretical model, for a target of $50 \% \mathrm{SL}_{\mathrm{t}}$ (that means zero SS) and for a target of $95 \% \mathrm{SL}_{\mathrm{t}}$. As shown in Fig. 2 the results are very different, in fact the gap between simulation outputs and theoretical expected values is relevant for a low $\mathrm{SL}_{t}$ target but this gap shrinks when the $\mathrm{SL}_{\mathrm{t}}$ target grows. According with the fact that EOQ model is often used with high SL target, it is possible to validate the simulation model independently from the used sequences ( $\mathrm{A}$ or $\mathrm{B}$ in this case). For the simulations the used sequence is B.

\subsection{Model Implementation and Experiments Set}

To prove the effectiveness of the proposed approach, the simulation experiments are developed to investigate the correlation between demand distribution and time sampling and expected SL. Demand is a normal distributed demand if the considered time sample is equal to a month and its shape is described by a minimum $\left(\mathrm{Min}_{\mathrm{d}}\right)$ and a maximum $\left(\mathrm{Max}_{\mathrm{d}}\right)$ value for the daily demand.

First the stochastic demand is generated, then the SS are calculated and the simulation model runs. Each simulation is performed with SS calculated but three different demand time sample are used to calculate $\sigma_{\mathrm{i}}$. Time sample is set equal to:

1. a month;

2. a month, with SS calculated according with $\sigma_{i}$ taken in the worst case;

3. the lead time, with SS calculated according with $\sigma_{\mathrm{i}}$ taken in the worst case.

In the first case demand time sample is set equal to the period of time used even for the stochastic generation of the demand, in other word, using this time sample the SS are calculated according with the imposed standard deviation used to generate the demand. In the second case the standard deviation $\sigma_{i}$ of the generated demand is calculated $\mathrm{N}_{\mathrm{p}}$ time and at each time the initial day of the period is translated by one. The SS are calculated according with the standard deviation $\sigma_{i}$ of worst case, so the actual value of $\sigma_{i}$ is considered. In the third case the time sample used is the lead time and the SS are calculated according with the worst possible case according with the procedure described above. The simulation results are given in terms of achieved SL and $\mathrm{SL}_{\mathrm{p}}$. The demand generation function is developed to be set with the parameters commonly available in practice. To characterize the stochastic demand the used parameters are: demand mean $\mathrm{D}_{\mathrm{i}}$ and $\sigma_{\mathrm{i}}$ demand standard deviation, both referred to a monthly time sample, maximum demand $\operatorname{Max}_{\mathrm{d}}$ experimented in a single day and minimum demand Mind experimented in a single day. The demand generation process acts as described below:

1. The demand $D_{i}$ is randomly generated for the i-period, according with the assumption of normal distribution period by period;

2. The $\mathrm{D}_{\mathrm{i}}$ period demand is divided into a defined number $\left(\mathrm{N}_{\mathrm{p}}\right)$ of single daily demand $\left(\mathrm{d}_{\mathrm{k}}\right)$ according with the following assumption:

a. $\mathrm{d}_{\mathrm{k}}$ must be higher than $\operatorname{Min}_{\mathrm{d}}$;

b. $\mathrm{d}_{\mathrm{k}}$ must be lower than $\mathrm{Max}_{\mathrm{d}}$; 
c. the $d_{k}$ daily demand are uniformly distributed between $\operatorname{Min}_{d}$ and $\operatorname{Max}_{\mathrm{d}}$;

d. the mean of the demand in the period is equal to $D_{i}$.

Table 2. Used parameters set

\begin{tabular}{lr}
\hline Parameter & Set value \\
\hline $\mathbf{D}_{\mathbf{i}}$ & $100.000,00$ \\
$\mathbf{N}$ & $10.000,00$ \\
$\mathbf{N p}$ & 20,00 \\
$\mathbf{C}_{\mathbf{l}}$ & 200,00 \\
$\mathbf{C}_{\mathbf{s}}$ & 1,00 \\
$\mathbf{L t}$ & 5,00 \\
$\boldsymbol{\sigma}_{\mathbf{t}}$ & 0,00 \\
$\mathbf{I m p o s e d} \mathbf{S L}$ & 0,95 \\
\hline
\end{tabular}

To investigate the influence of $\operatorname{Min}_{d}$ and $\operatorname{Max}_{d}$ at different level of $\sigma_{i}$ all the other parameters of the model are set to specific values. The simulation model parameters are set in order to be representative for small-medium manufactures operating in the food sector. The used set is illustrated in table 2. The experiments investigate the various combination between $\operatorname{Min}_{\mathrm{d}}$ and $\mathrm{Max}_{\mathrm{d}}$ at different level of $\sigma_{\mathrm{i}}$. The experiments were performed to investigate the range of $\operatorname{Min}_{d}$ between 0 to $\mathrm{D}_{\mathrm{i}} / 2 * \mathrm{~N}_{\mathrm{p}}$ (in this case from 0 to 2.500 units, with a step of 500 units) and the range of $\mathrm{Max}_{\mathrm{d}}$ from $\mathrm{D}_{\mathrm{i}} / \mathrm{N}_{\mathrm{p}}$ to $\mathrm{D}_{\mathrm{i}}$ (in this case from 5.000 to 100.000 units, with a step of 2.500 units). The demand monthly standard deviation $\sigma_{i}$ varies from 0 up to the $20 \%$ of $D_{i}$ (in this case from 0 to 20.000 units, with a step of 5.000 units), in order to simulate a scenario of long term demand stability and a scenario of long term demand fluctuation.

\section{$4 \quad$ Findings}

The presented experiment implies a number of 1.170 simulations, each of them is performed three times according with the choose time sample to evaluate $\sigma_{i}$ and the consequent SS value, and each simulation provide two results: achieved SL and $\mathrm{SL}_{\mathrm{p}}$. The results can be represented with diagrams as shown in Fig. 3. The generated demands $D_{i}$ were analyzed to check the assumptions described in chapter 3.2. The assumptions: $\mathrm{a}, \mathrm{b}$ and $\mathrm{c}$ are always respected while the assumption $\mathrm{d}$ is not always guarantee. A level of $98 \%$ of $\mathrm{D}_{\mathrm{i}}$ is undertaken to accept the results of the experiments. According with this threshold value, it is possible to observe that the assumption is not respected when the maximum value admitted for Max $_{d}$ is small, lower than $12,5 \%$ of $\mathrm{D}_{\mathrm{i}}$. This area is not very interesting because under these conditions the model acts as the standard EOQ model. The imposed value of $\sigma_{i}$ (considering the worst case using a time sample of a month) is respected only for the simulation with a low admitted $\operatorname{Max}_{\mathrm{d}}$. In all the other cases the variables $\operatorname{Min}_{\mathrm{d}}$ and $\operatorname{mostly} \mathbf{M a x}_{\mathrm{d}}$ drive the actual value of $\sigma_{i}$ that became almost independent from the initial imposed value, see Fig 3. The results show how the achieved service level is significantly lower than the imposed value of $95 \%$ and the behavior of SL and $\mathrm{SL}_{\mathrm{p}}$ is similar. 

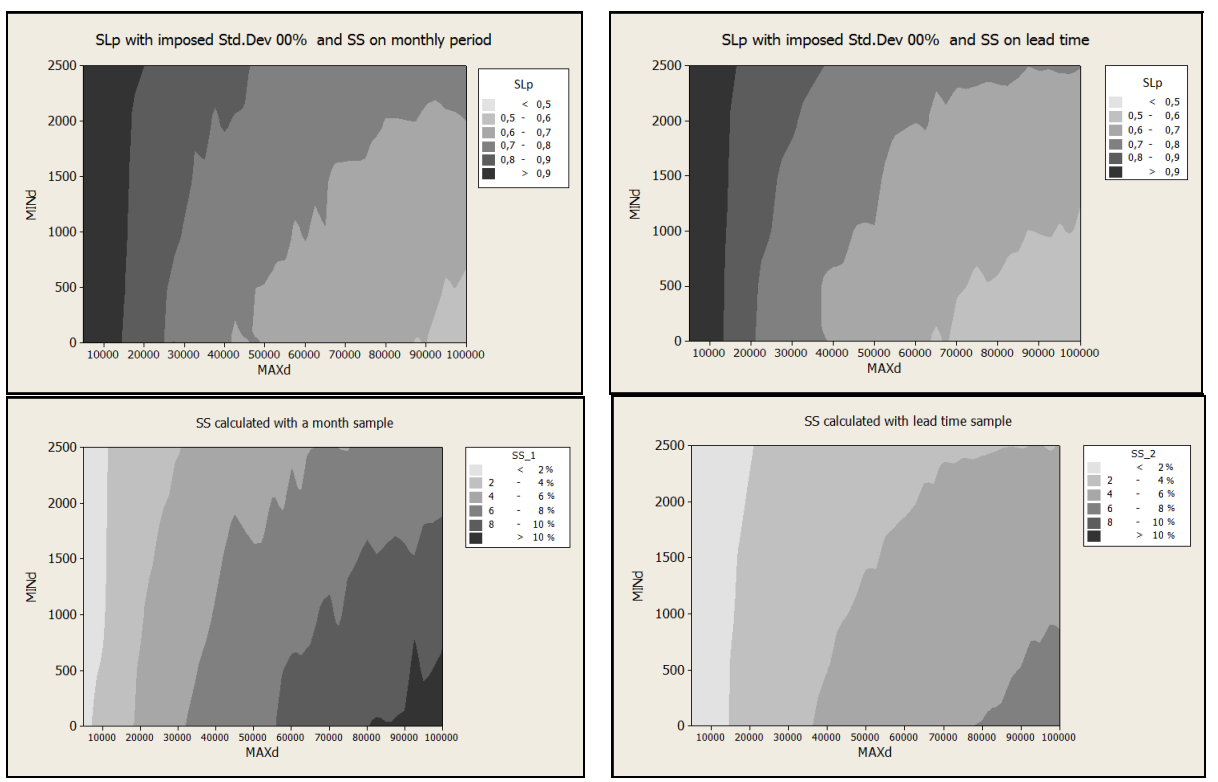

Fig. 3. Achieved service level $\mathrm{SL}_{\mathrm{p}}$ and related $\mathrm{SS}$ for $\sigma_{\mathrm{i}}=0$ under relaxed assumption using different time sample (month on the left and lead time on the right)
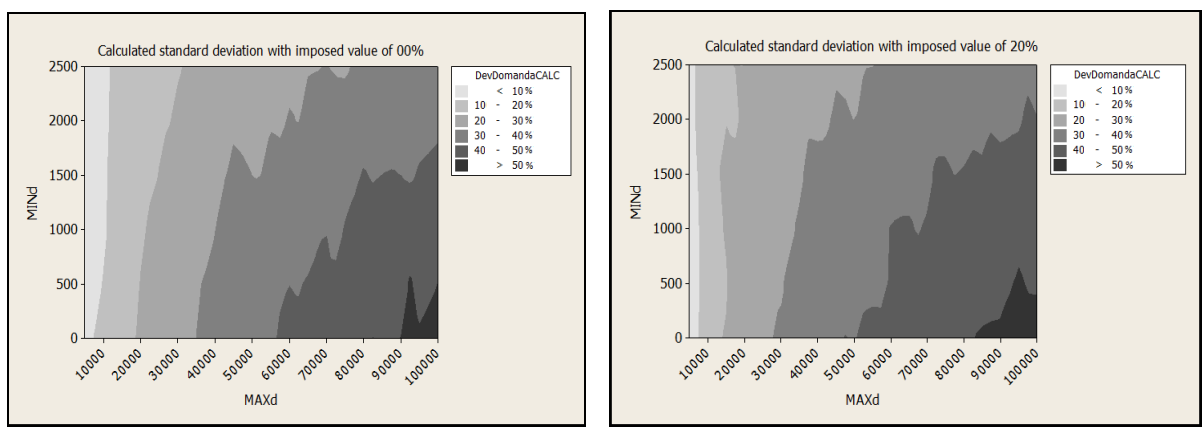

Fig. 4. Actual values of $\sigma_{\mathrm{i}}$ in simulations at different values of imposed for $\sigma_{\mathrm{i}}$ : $00 \%$ and $20 \%$

\section{Conclusions}

The present work demonstrates that discrete event simulation approach can be useful to investigate EOQ model behavior under relaxed assumptions. Simulation technique can be useful to extend a mathematical continuous model even thought validation process has to be handled carefully because of discretization problems. In the presented example this approach is implemented to study the correlation between demand shape, sampling problems and achieved SL. The results show that when demand normal distributed hypothesis is removed the complexity of the EOQ model becomes very high and experimenters has to face different problems such as: how to 
describe demand shape and sampling problems. Moreover discrete event simulation model gives outputs for a specific parameters set and this prevents to enlarge automatically the results.

Although the previous considerations, the simulation experiments reveal that under relaxed demand assumptions the EOQ model performances are significantly different from theory. The achieved service level (SL) is significantly lower than the imposed value (in this case 95\%). The expected SL depends on demand lumpiness that in these experiments is characterized by $\operatorname{Max}_{\mathrm{d}}, \mathrm{Min}_{\mathrm{d}}$ and $\sigma_{\mathrm{i}}$. The results shows that the expected SL is strongly related to the maximum demand $\mathrm{Max}_{\mathrm{d}}$ admitted in a single day. High values of SL can be observed when $\mathrm{Max}_{\mathrm{d}}$ is small, in these cases the model behavior is very similar to standard EOQ model. In general the achieved SL falls when $\mathrm{Max}_{\mathrm{d}}$ grows and this effect is grater at $\operatorname{small}_{\mathrm{Min}} \mathrm{d}_{\mathrm{d}}$ values, when the minimum demand admitted in a single day is close to zero. The periodic demand standard deviation $\sigma_{i}$ seems to have a small influence on SL. Comparing a scenario with low $\sigma_{i}$ with a scenario with high $\sigma_{\mathrm{i}}$ the results show a slight reduction for the achieved SL associated with an increase of the safety stocks SS. This SL reduction is appreciable for small values of $\mathrm{Max}_{\mathrm{d}}$ and for high values of $\mathrm{Min}_{\mathrm{d}}$, when demand lumpiness is low. About the time sample used to calculate $\sigma_{i}$, and $\mathrm{SS}$, results show that, even with demand relaxed assumptions, a time sample equal to the lead time assures a good trade-off between SL and SS.

\section{References}

1. Harris, F.W.: Howmany parts to make at once. Factory, The Magazine of Management 10(2), 135-136, 152 (1913), reprinted in Operations Research 38(6) (November-December 1990)

2. Brown, R.G.: Smoothing, Forecasting and Prediction of Discrete Time Series. PrenticeHall, Englewood Cliffs (1963)

3. Bather, J.A.: A continuous time inventory model. J. Appl. Prob. 3, 538-549 (1966)

4. Tersine, R.J.: Principles of inventory and materials management. North-Holland, Elsevier Science Publishing Co., Inc. (1988)

5. Ghiani, G., Laporte, G., Musmanno, R.: Introduction to logistics systems planning and control. John Wiley \& Sons Ltd., West Sussex (2004)

6. Markland, R.E., Vickery, S.K., Davis, R.A.: Operation management, 2nd edn. SouthWestern, Ohio (1998)

7. Humphrey, A.S., Taylor, G.D.: Stock level determination and sensitivity analysis in repair/rework operations. Int. J. Op. \& Prod. Man. 18(6), 612-630 (1998)

8. Borgonovo, E.: Sensitivity analysis with finite changes: An application to modified EOQ models. Eu. J. Op. Res. 200, 127-138 (2010)

9. Chan, W.M., Ibrahim, R.N., Lochert, P.B.: A new EPQ model: integrating lower pricing, rework and reject situations. Prod. Plan. \& Cont. 14(7), 588-595 (2003)

10. Goyal, S.K., Giri, B.C.: Recent trends in modeling of deteriorating inventory. Eu. J. Opl. Res. 134, 1-16 (2001)

11. Ferguson, M., Jayaraman, V., Souza, G.C.: Note: Application of the EOQ model with nonlinear holding cost to inventory management of perishables. Eu. J. Opl. Res. 180, 485490 (2007) 
12. Inderfurth, K., Linder, G., Rachaniotis, N.P.: Lot sizing in a production system with rework and product deteriorating. Int. J. Prod. Res. 43(7), 1355-1374 (2005)

13. Chao, H.: The EOQ model with stochastic demand and discounting. Eu. J. Op. Res. 59, 434-443 (1992)

14. Lowe, T.J., Schwarz, L.B.: Parameter estimation for the EOQ lot-size model: minimax and expected value choices. Naval Res. Log. Quart. 30, 367-376 (1983)

15. Hojati, M.: Bridging the gap between probabilistic and fuzzy-parameter EOQ models. Int. J. Prod. Ec. 91, 215-221 (2004)

16. Dobson, G.: Sensitivity of the EOQ model to parameter estimates. Op. Res. 36(4), 570574 (1988)

17. Mykytka, E.F., Ramberg, J.S.: On the sensitivity of the EOQ to errors in the forecast of demand. IIE Trans. 16(2), 144-151 (1984)

18. Mahmoud, M.S., Younis, M.A.: Sensitivity analysis of productive inventories under modeling errors. Mat. Comp. Mod. 13(7), 65-75 (1990)

19. Higle, J.L.: A note on the sensitivity of the EOQ. IIE Trans. 21(3), 294-297 (1989)

20. Mehrez, A., Ben-Arieh, D.: All-unit discounts, multi-item inventory model with stochastic demand, service level constraints and finite horizon. Int. J. of Prod. Res. 29(8), 1615-1628 (1991)

21. Naddor, E.: Sensitivity to distributions in inventory systems. Man. Sci. 24, 1769-1771 (1978)

22. Hebert, J.E., Deckro, F.: A simulation analysis of dynamic inventory policies in a generalized stochastic environment. In: Proc. of the W. Sim. Conf., Washington, DC, pp. 843-847 (December 1977)

23. Benton, W.C.: Safe stock and service levels in periodic review inventory system. J. Opl. Res. Soc. 42(12), 1087-1095 (1991)

24. Lau, H.S., Zaki, A.: The sensitivity of inventory decisions to the shape of lead time demand distribution. IIE Trans. 14, 265-271 (1982)

25. Ziegler, R.: Criteria for measurement of the cost parameters of an economic order quantity. Ph.D. dissertation, Univ. of North Carolina at Chapel Hill (1973)

26. De Castro, E., Tabucanon, M.T., Nagarur, N.N.: A production order quantity model with stochastic demand for a chocolate milk manufacturer. Int. J. Prod. Ec. 49, 145-156 (1997)

27. Persona, A., Battini, D., Manzini, R., Pareschi, A.: Optimal safety stock levels of subassemblies and manufacturing components. Int. J. Prod. Eco. 110, 147-159 (2007) 